\title{
Appetite Dysregulation and the Apelinergic System are Connected to the Global Chronic Disease Epidemic
}

\author{
Martins IJ ${ }^{1,2,3,4^{*}}$ \\ ${ }^{1}$ School of Medical Sciences, Edith Cowan University, Australia \\ ${ }^{2}$ Centre of Excellence in Alzheimer's Disease Research and Care, Sarich Neuroscience Research Institute, Edith \\ Cowan University, Australia \\ ${ }^{3}$ School of Psychiatry and Clinical Neurosciences, The University of Western Australia, Australia \\ ${ }^{4}$ McCusker Alzheimer's Research Foundation, Hollywood Medical Centre, Australia
}

*Correspondence: Ian James Martins, School of Medical Sciences, Edith Cowan University, Australia

Received on 30 December 2019; Accepted on 06 January 2020; Published on 10 January 2020

Copyright (C) 2020 Martins IJ. This is an open access article and is distributed under the Creative Commons Attribution License, which permits unrestricted use, distribution, and reproduction in any medium, provided the original work is properly cited.

Keywords: apelinergic, appetite control, apelin, sirtuin 1, nutritional therapy, global, mitophagy, aging, diabetes, chronic disease

\section{Editorial}

The apelinergic system and its relevance to endocrinology and diabetes has become of major importance to the global chronic disease epidemic. Apelinergic dysregulation is associated with appetite dysregulation [1] and connected to insulin resistance and various organ diseases that include the heart, adipose tissue, liver and the brain. The peptide apelin and its receptor APJ (G protein-coupled receptor) play an important role in various tissues and the role in stress, insulin resistance, hormone release, thermoregulation, food intake and water balance are critical to endocrinology and diabetes (Figure 1). Recent studies have indicated that apelin is now identified as an anti-aging factor and its inactivation leads to a decline in the apelinergic axis [2] with accelerated aging and programmed cell death that is linked to endocrinology, diabetes and neurodegenerative disease with relevance to the global chronic disease epidemic [3-6]. Apelin regulates the aging process by interactions with sirtuins [3] and its regulation of sirtuins 1-7 is critical in the prevention of the accelerated aging process and chronic disease.

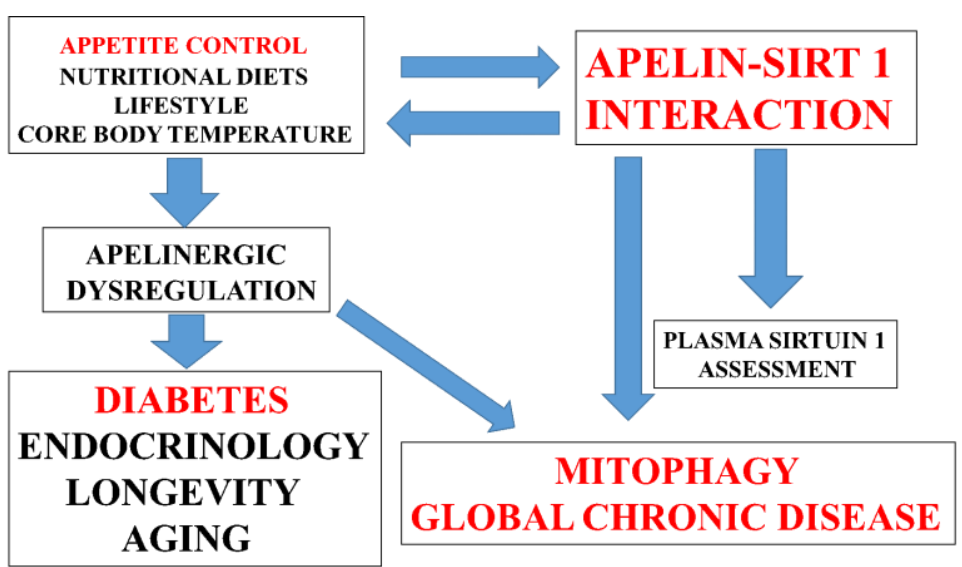

Figure 1: Appetite control, dietary interventions, lifestyles and core body temperature are linked to apelinergic dysregulation in endocrinology and diabetes with relevance to mitophagy and global chronic disease epidemic. 
Sirtuin 1 (SIRT1) has now been identified as an anti-aging gene [7] and apelin dysregulation may lead to downregulation of the SIRT1 gene with accelerated programmed cell death. Apelin and SIRT1 are linked to the central co-ordination and homeostasis of the neuroendocrine system that are controlled by lifestyle, diet, external stressors and appetite dysregulation (apelin-SIRT1 defect) that may lead to the acceleration of the global chronic disease epidemic. The apelin- SIRT1 dysregulation is connected to nitric oxide disturbances that induce diabetes, endocrine, metabolic and mental disorders $[1,8,9]$. Regulation of SIRT1 is now critical to age related diseases and longevity control with SIRT1 regulation of mitochondrial apoptosis linked to endocrinology and global chronic disease [1015]. Apelin regulation of sirtuins has now become of major research interest with apelin control of SIRT1 linked to the cellular SIRT2-7 with relevance to mitophagy and programmed cell death (Figure 1). The discovery of SIRT1 as the heat shock gene $[16,17]$ may now be relevant to apelin-SIRT1 role in thermoregulation and appetite control relevant to endocrinology and diabetes.

Nutritional regulation and appetite control are essential to maintain the apelinergic axis and the apelin-SIRT1 interaction now relevant to the global chronic disease epidemic. Activators and inhibitors [18] of SIRT1 should be carefully assessed with relevance to apelinergic activation and dysregulation of the aging process and longevity. The release of the anti-aging protein SIRT1 in the blood plasma [19] should be assessed to determine an intact apelinergic axis with relevance to apelin and its role as an anti-aging factor in endocrinology and diabetes (Figure 1). Nutritional diets are associated with apelin-SIRT1 regulation of amyloid beta metabolism [1, 20, 21] that are connected to the anti-aging processes and Alzheimer's disease with food quality critical to maintain the apelin-SIRT1 interaction and the prevention of Alzheimer's disease and neurological diseases [22].

\section{Acknowledgement}

This work was supported by grants from Edith Cowan University, the McCusker Alzheimer's Research Foundation and the National Health and Medical Research Council.

\section{References}

1. Martins IJ. Nutritional diets accelerate amyloid beta metabolism and prevent the induction of chronic diseases and Alzheimer's disease. Photon ebooks. 2015.

2. Rai R, Ghosh AK, Eren M, et al. Downregulation of the apelinergic axis accelerates aging, whereas Its systemic restoration improves the mammalian healthspan. Cell Rep. 2017;21(6):1471-80.

3. Zhou Q, Chen L, Tang M, et al. Apelin/APJ system: A novel promising target for anti-aging intervention. Clin Chim Acta. 2018;487(1):233-40.

4. Martins IJ. Nutritional and genotoxic stress contributes to diabetes and neurodegenerative diseases such as Parkinson's and Alzheimer's Diseases. In: Atta-ur-Rahman, ed. Frontiers in Clinical Drug Research -CNS and Neurological Disorders. 3rd ed. Sharjah: Bentham Science Publishers; 2015. p. 158-192.

5. Martins IJ, Calderón AM. Diet and nutrition reverse Type 3 diabetes and accelerated aging linked to global chronic diseases. J Diab Res Ther. 2016;2(2):1-6.

6. Martins IJ. Insulin therapy and autoimmune disease with relevance to non-alcoholic fatty liver disease. An Update. IntechOpen. 2018.

7. Martins IJ. Anti-aging genes improve appetite regulation and reverse cell senescence and apoptosis in global populations. Advances in Aging Research. 2016;5(1):9-26. 
8. Martins IJ. Apelinergic system defects with relevance to mental disorders in diabetes. World J Psychiatry Ment Health Res. 2017;1(1):1-3.

9. Martins IJ. Apelin and Sirtuin 1 dysregulation induce endocrine and metabolic disorders in chronic disease. Glob J Endocrinol Metab. 2017;1(1):1-2.

10. Kordala A, Pietrzak B. Does SIRT-1 mediate calorie restriction and prolong life? - A mini review. Pol J Food Nutr Sci. 2014;64(3):159-64.

11. Yang T, Fu M, Pestell R, et al. SIRT1 and endocrine signaling. Trends Endocrinol Metab. 2006;17(5):186-91.

12. Lavu S, Boss O, Elliott PJ, et al. Sirtuins--novel therapeutic targets to treat age-associated diseases. Nat Rev Drug Discov. 2008;7(10):841-53.

13. Imai S, Guarente L. It takes two to tango: NAD+ and sirtuins in aging/longevity control. NPJ Aging Mech Dis. 2016;2:16017.

14. Pereira CV, Lebiedzinska M, Wieckowski MR, et al. Regulation and protection of mitochondrial physiology by sirtuins. Mitochondrion. 2012;12(1):66-76.

15. Martins IJ. Genomic medicine and endocrine autoimmunity as key to mitochondrial disease. Glob J Endocrinol Metab. 2018;2(2):1-3.

16. Martins I.J. (2019) Body temperature regulation determines immune reactions and species longevity. In: Asea A., Kaur P. (eds) Heat shock proteins in neuroscience. Heat Shock Proteins, vol 20. Springer, Cham.

17. Martins IJ. Heat shock gene inactivation and protein aggregation with links to chronic diseases. Diseases. 2018;6(2):1-5.

18. Martins IJ. Nutrition therapy regulates caffeine metabolism with relevance to NAFLD and induction of type 3 diabetes. J Diabetes Metab Disord. 2017;4(1):1-9.

19. Martins IJ. Sirtuin 1, a diagnostic protein marker and its relevance to chronic disease and therapeutic drug interventions. EC Pharmacology and Toxicology. 2018;6(4):209-15.

20. Masoumi J, Abbasloui M, Parvan R, et al. Apelin, a promising target for Alzheimer disease prevention and treatment. Neuropeptides. 2018;70(1):76-86.

21. Aminyavari S, Zahmatkesh M, Farahmandfar M, et al. Protective role of Apelin-13 on amyloid $\beta 25-35$ induced memory deficit; Involvement of autophagy and apoptosis process. Prog Neuropsychopharmacol Biol Psychiatry. 2019;89(1):322-34.

22. Martins IJ. Food quality induces a miscible disease with relevance to Alzheimer's disease and Neurological diseases. J Food Research. 2016;5(6):45-52. 